\title{
A NEW APPROACH FOR DOCUMENTATION, CONSERVATION AND PRESERVATION OF THE MONOLITHIC STATUES IN SAN AGUSTÍN AS PILOT FOR OUTDOOR CULTURAL HERITAGE DOCUMENTATION IN COLOMBIA
}

\author{
A. Estela ${ }^{\mathrm{a}, *}$, J. Hamacher ${ }^{\mathrm{b}}$ \\ ${ }^{a}$ Research Associate, ICANH (Instituto Colombiano de Antropología e Historia), Calle 12 No. 2-41 Bogotá D.C., Colombia - \\ aestela@hotmail.com \\ ${ }^{\mathrm{b}}$ PhD Candidate, AEGIS-CEMA-INCAL, Université catholique de Louvain, Place Blaise Pascal, 1, Bte L3.03.13, B-1348, Louvain-la- \\ Neuve, Belgium - julian.hamacher@gmail.com
}

KEY WORDS: San Agustín, Mantis, Z+F, Outdoor Heritage, ICANH, Monolithic Statues, Post Processing

\begin{abstract}
:
The "Instituto Colombiano de Antropología e Historia" (ICANH) started a new conservation project for the "San Agustín Archaeological Park" (Huila, Colombia) in 2013. The objectives of this project are the documentation, conservation, and preservation of the numerous monolithic statues mainly by integrating the use of new technologies ( $3 \mathrm{D}$ models). A first phase of the project has been completed, resulting in three-dimensional models of 66 of the monolithic sculptures in San Agustín. The methodology developed in this first phase will show the way for other heritage sites in Colombia and for subsequent phases applied to the archaeological park. The 3D data has been obtained using two types of data acquisition technology: the Mantis Vision F5 using infrared structured-light (SL) and a laser scanner based on the phase shift (PS) technology, the Z+F Imager 5010. The results show that future phases need improvement in data acquisition. Mainly the data obtained with the hand held scanner shows many lacunae. This article presents the observations during data processing on the basis of one sculpture, "Escultura 23". In conclusion, this first phase showed where to improve for the succeeding ones, for instance the detail of the meshes need to be increased if the models are to be used for detailed conservation and preservation purposes.
\end{abstract}

\section{THE PROJECT}

The open air archaeological park of "San Agustín" (Huila, Colombia) figures on the UNESCO's World Heritage List since 1995. The area is home to numerous monolithic statues belonging to funerary complexes that date back to the "Clásico Regional" period (1st- 10th century AD). In February 2013, the ICANH started a new conservation programme for this important heritage site including the use of the latest technologies of documentation. This project is intended as a pilot project for the whole country by applying 3D technology in the field of conservation, preservation and documentation. Creating three-dimensional models for outdoor heritage monuments is a total novelty in Colombia and to date even in scientific literature similar projects concerning monolithic statues with this kind of approach are seldom. The methodology developed in this first phase will show the way for other heritage sites in Colombia and it aims at showing the advantages of a virtual three-dimensional approach to heritage.

In this manner, the project is carried out in various phases and so far only the first consisting in the data acquisition with the subsequent $3 \mathrm{D}$ modelling has been completed and gave birth to the digital models of a total of 66 statues found in the so called "Mesitas" A and B as well as in the "Bosque de las Estatuas" (with an additional model of the "Fuente del Lavapatas"). The next steps will be to demonstrate in how far the 3D models can be used for educational, conservation and preservation purposes. For instance the digital models have already been showcased in during an exhibition in Bogota. More than showing the digital models to a large public, we hope to be able to associate the models to conservational and archaeological data in order to render research easier and increase the accessibility to the artefacts.

The implementation of new non-destructive technologies for the study of cultural heritage in countries like Colombia still faces various difficulties today and progresses only slowly. One main factor is the lack of knowledge of those new technologies and that only a few specialists could bring in their expertise for projects such as this in order to obtain satisfactory results. Another factor is the lack of equipment for data acquisition. Only few companies, generally resellers of the most renowned brands, have such equipment and offer to rent them with an operator who is not acquainted with scanning cultural heritage. The planning and progress of this project has been highly influenced by both of the upper mentioned factors. The lack of discussion about the data with experts, regarding many of the points addressed here below, would have greatly helped in obtaining more conclusions and certainly better results.

\section{USED EQUIPMENT}

The 3D data has been obtained using two types of data acquisition technologies: the Mantis Vision F5 using infrared structured-light (SL) and a laser scanner based on the phase shift (PS) technology, the Z+F Imager 5010. It is difficult to compare them as they use a very different approach of acquiring $3 \mathrm{D}$ data. The Mantis can be used directly on the surface of an object and should not be held further away than $1 \mathrm{~m}$. The $\mathrm{Z}+\mathrm{F}$ has a minimal distance of $0.3 \mathrm{~m}$ and could theoretically be used up to a distance of about $183 \mathrm{~m}$ losing then obviously much accuracy. The maximal acquisition speed for the Imager 5010 is defined at about 1 million points per second whereas the F5 has a fix

\footnotetext{
* Corresponding author.
} 
acquisition speed of 0.5 million points per second. For the Mantis, the accuracy at $1 \mathrm{~m}$ is up to $0.5 \mathrm{~mm}$ and for the $\mathrm{Z}+\mathrm{F}$ up to $0.6 \mathrm{~mm}$ at $10 \mathrm{~m}$.

The photographs for the textures of the models have been taken using a Canon EOS 40D using an EF 24-70 f:2.8 lens.

For the geo-referencing of the statues, a GNSS receiver Javad Triumph-1 has been employed which uses 216 channels and can receive different signals (GPS, L1, L2, L2C Y L5, GLONAS L1 and L2). (1)

So far, for the theoretical abilities of the equipment used, in practical terms, a selection of sculptures has been scanned with both scanners in order to enable a comparison of the results. However, the main part of the models has been obtained by using the Z+F Imager 5010. The Mantis has been used mostly where the accessibility of the monuments was difficult due to vegetation or other static objects located close to the target to be scanned. Indeed, those constraints made the use of the $\mathrm{Z}+\mathrm{F}$ sometimes impossible. The minimal distance could not be respected, thus leaving no choice but using the hand-held scanner to obtain results. Furthermore, the simple fact that all scanned objects are located outdoor had a critical impact on lighting conditions simply because they could not be controlled. In addition, some of the monuments are protected against weather conditions by roofs set up on simple pilasters which obviously cast shadows. Some of the statues are even located under a baldachin like structure of massive stone. Indeed, the choice of the two different scanning systems was based upon the above described location of the sculptures.

\section{WORKFLOW}

1) Data acquisition with the $\mathrm{Z}+\mathrm{F}$ Imager 5010 using targets for easier alignment. In the meantime, the GPS data have been taken using the Javad Triumph-1.

2) Data acquisition with the Mantis Vision F5 (where it was to be used).

3) Taking the photographs for colour texturing with a Canon EOS 40D. Eight photos have been made for each sculpture trying to avoid shadows on the subject and shooting with the most "uniform" light on the subject.

4) Processing of the point clouds in Cyclone and Mantis Vision's Production (MVP).

5) Alignment of the point clouds in PolyWorks and Geomagic Studio.

6) Creation of the models with PolyWorks, MeshLab and Geomagic Studio.

7) Texturing with MeshLab.

8) Saving the models in PLY format to guarantee accessibility of the models.

\footnotetext{
${ }^{1}$ All equipment specific data have been taken from official data sheets from the respective manufacturer.
}

\section{DATA ACQUISITION AND POST PROCESSING}

After the export of the data via the scanner specific software solutions, the point clouds have been elaborated and aligned in PolyWorks as well as in Geomagic Studio and later visualized also with MeshLab. During this phase of work many issues came to light that are rather to be linked to the previous phase (data acquisition).

The most obvious of those problems was the lack of points that many of the models showed on similar parts of the statues, mainly the lateral faces. Indeed, throughout the models many show lacunae on their sides whether they have been taken with the $\mathrm{Z}+\mathrm{F}$ or with the Mantis. In general, we can affirm that it is difficult to scan the statues that are made of quite flat stone blocks, as for example "Escultura 23" (see infra for a more detailed discussion about this statue). Also, quite curvy parts of the statues seem generally to be more difficult to scan using the Mantis because one needs to pay attention to the angle at which the light hits the target to obtain detailed frames. Also, we determined a major point density obtained with the Mantis. For some of the statues, the number of points obtained with the hand held scanner was ten times higher than the number obtained by the terrestrial based scanner (see case study - infra). This is in part due to the high number of frames that have been taken and which often interfere with each other and superpose themselves. The big quantity of frames, often exceeding 300, and their overlapping resulted in difficulties when properly aligning the data from the Mantis and hence made the creation of a good mesh no easy matter. Indeed, this fact diminishes the purely practical advantage of the handheld scanner since the raw data from the $\mathrm{Z}+\mathrm{F}$ was easier to align and to transform into a mesh. The difficulty of a correct alignment of such an elevate number of frames with the potential deviations was the main reason when choosing to primarily use data from the $\mathrm{Z}+\mathrm{F}$ to create the majority of the models.

As to the meshes, usually, they represent the general form of the statues but often do not show the fine relief of the stone surface which in most cases becomes visible only when the texture is applied to the model. Surely, the detail could have been higher accounting for a better data acquisition with both the Mantis and the $\mathrm{Z}+\mathrm{F}$.

\section{CASE STUDY - "ESCULTURA 23" (MESITA B)}

"Escultura 23" is a monolithic anthropo-zoomorphic sculpture in triangular form located in the "Mesita B". The carved stone block is in its overall form quite flat. The most protruding part of the relief is the nose. The cheeks are each represented by circular shapes that protrude much less than the nose. The mouth is of oval form with thick lips that are open and let see two rows of small teeth with however big triangular canines. Their eye sockets are carved quite deeply in the stone and are of semicircular form, each containing a perfect circular eyeball. On the broad forehead, the sculpture bears a carved line which might indicate the separation between skin and hair or maybe a kind of adornment. Particular of this case study, and also for the other sculptures at San Agustín, is the low contrast that these objects present with a stone surface varying for each object little in colour.

According to Sotomayor and Uribe (Sotomayor and Uribe, 1987), this statue has been found (fig. 1) in front of the north- 
western mound where Pérez de Barradas (Pérez de Barradas, 1943) was excavating and found obsidian chips, red ceramics decorated with black lines and a sepulture. The famous Italian explorer Codazzi states in his "Antiguedades indigenas. Ruinas de San Agustín" that behind this sculpture (number 23) was located the sculpture number 24 and several gravestones with incised motives on their inner faces.

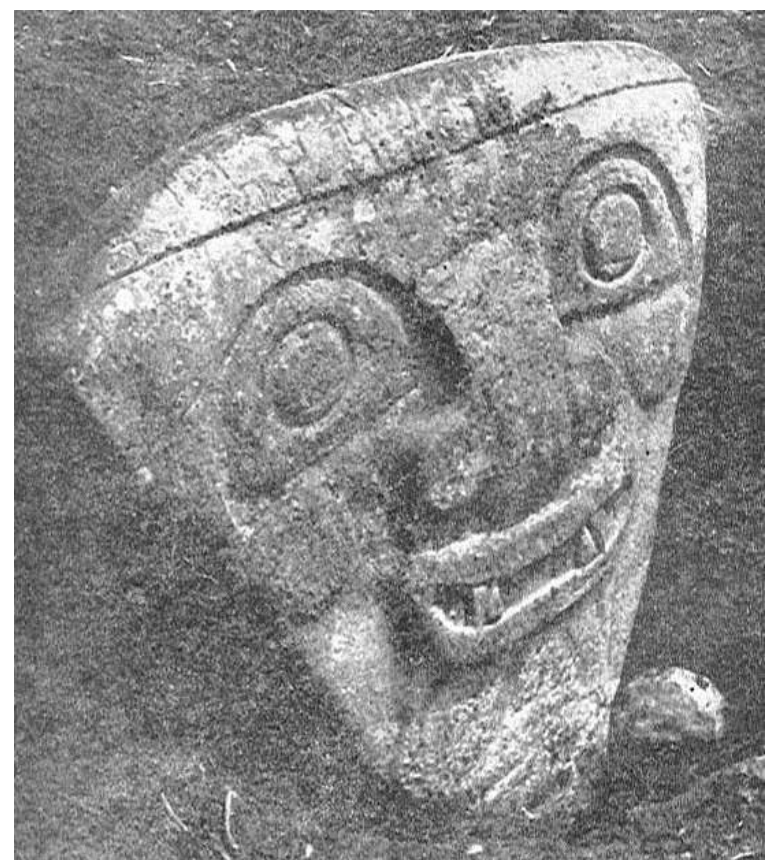

Figure 1. "Escultura 23". Photograph from 1943. (Pérez de Barradas, 1943, table 72).

For "Escultura 23", the lower part, where the statue must have been buried, shows more ochre tones mixed to the overall grey of the tuff stone. Interesting is the marked dark brown line touching the upper lips and the lower part of the nose which was most probably provoked by the stagnating humidity on ground level when the bottom was laying beneath the surface. The low contrast of the object with though many carved parts and uncontrolled light conditions resulted in shadows on the sculpture when scanning. Furthermore the data acquisition was rendered more difficult due to the rear bottom part being still covered by terrain (fig. 1).

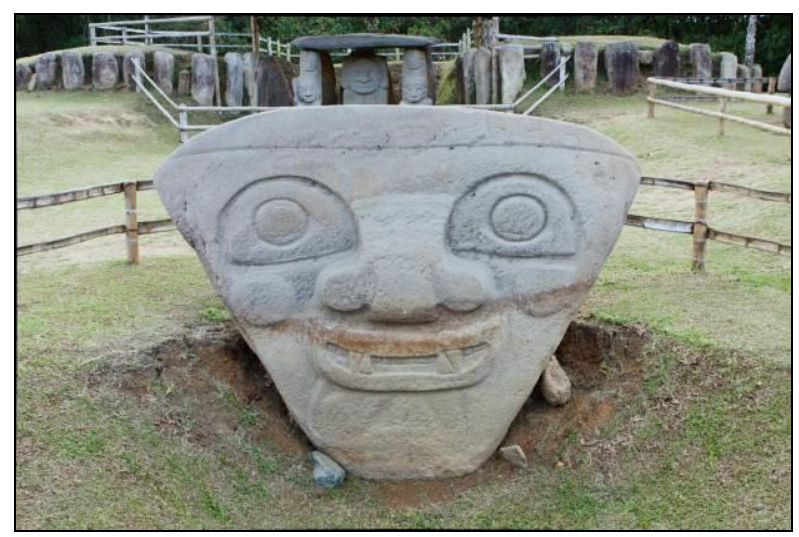

Figure 2. Photo of "Escultura 23" showing the fence and the rear part still being covered by the terrain.
Similarly, the fence around the object made a proper positioning of the $\mathrm{Z}+\mathrm{F}$ when scanning the rear part tricky and favoured the use of the Mantis.

As already observed in general, the density of the point cloud emanating from the $\mathrm{Z}+\mathrm{F}$ was lower than the one from the Mantis. In this manner the cleaned point cloud from the $\mathrm{Z}+\mathrm{F}$ showed a total of ca. 1 million points whereas the Mantis produced a much denser one counting for ca. 8.8 million points. At this stage, we do believe that a proper handling of the $\mathrm{Z}+\mathrm{F}$ scanner (as well as waiting for better lighting conditions) would have resulted in a denser point cloud. The raw data for the $\mathrm{Z}+\mathrm{F}$ has been taken in 4 different scans whereas there was a total of 420 frames from the Mantis. We noted that the Mantis showed significantly more issues when scanning dark shadowy zones and fortunately the shadows of the fence did not fall as far as to reach the sculpture itself (fig. 2).

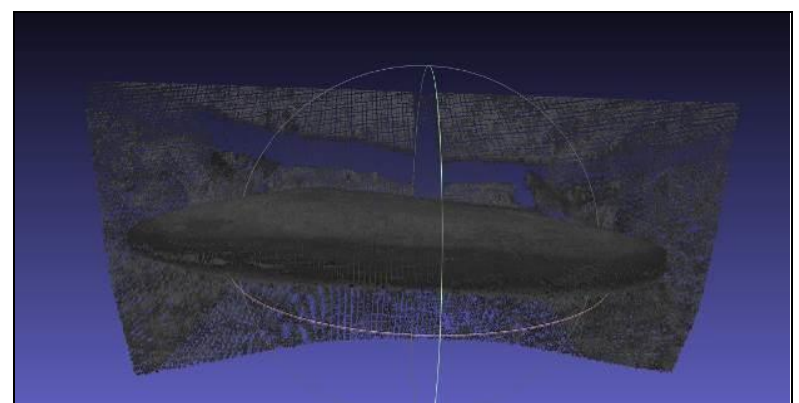

Figure 3. "Escultura 23". Mantis point cloud with shadow areas resulting in very low point density.

Both scanners showed nonetheless issues at the angles of the monument (fig. 4 and 5) resulting in very low point density for those parts, if none in the absence of points.
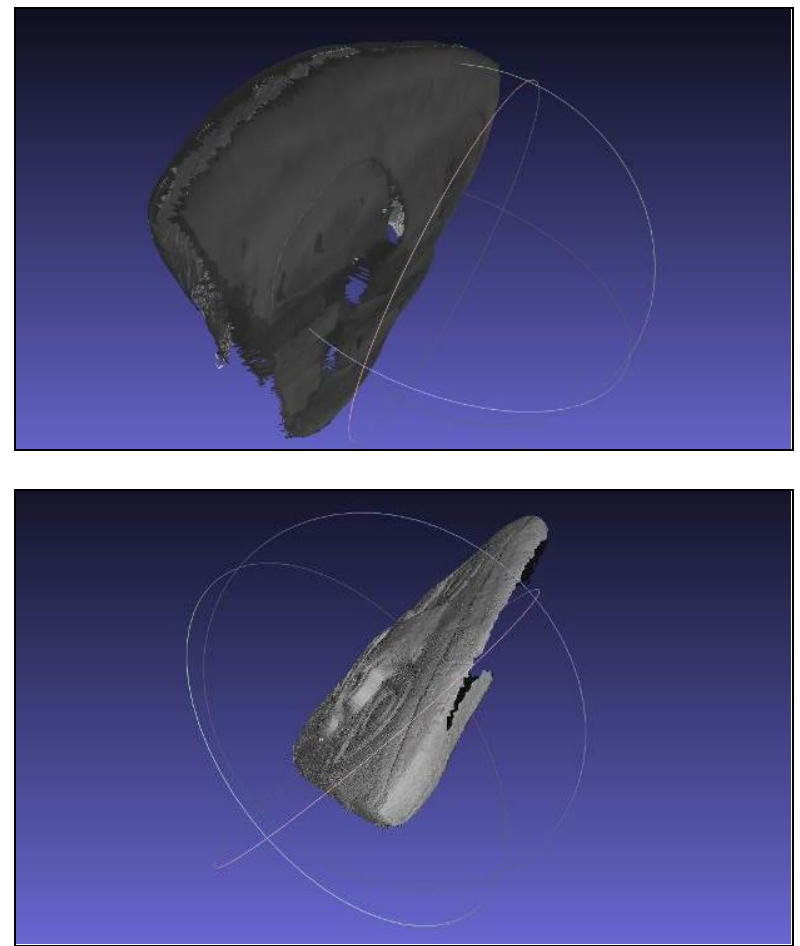

Figure 4 and 5. "Escultura 23". Meshes showing the lack of points/polygons on the angles of the monument. 
These lacunae were very visible for the $\mathrm{Z}+\mathrm{F}$ (fig. 4) and to a lesser extent for the Mantis which lacked data mainly on the upper part of the sculpture (fig. 5) and in the centre of the rear part. The latter could probably have been avoided by using a more elevated position when scanning. This issue with "Escultura 23" is representative of a number of other models. As we mentioned before, this holds true especially for the sculptures made out of a quite flat stone block (fig. 6).

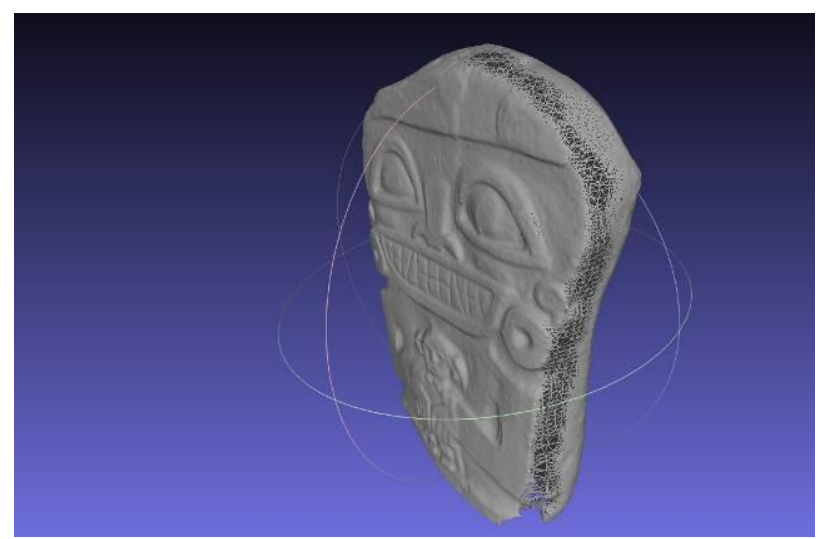

Figure 6. "Escultura 202". Missing points on the lateral parts and on the top.

For this case study, both point clouds from the Mantis and from the $\mathrm{Z}+\mathrm{F}$ have been elaborated in Geomagic Studio and MeshLab. The idea behind this approach was to try in succeeding to merge the meshes from both scanners and to determine the deviation between them.

In this manner, we imported each frame from the Mantis in Geomagic Studio which aligned them well. Subsequently, we cleaned the point cloud so as to leave only the points representing the actual statue counting for a total of about 9 million. The different frames were transformed into a mesh and combined resulting in ca. 17.5 million triangles- which we later decimated to ca. 2.5 million points. The same process has been undertaken for the 4 different point clouds emanating from the $\mathrm{Z}+\mathrm{F}$ which had a total of about 1 million points. The transformation into polygons has been done with the same settings as for the frames from the Mantis (no limit for max. triangle count). The result was a mesh counting for 1.9 million triangles. Tough the algorithm had issues when converting the points at the left side of the nose (from visualization viewpoint) into polygons (fig. 7).

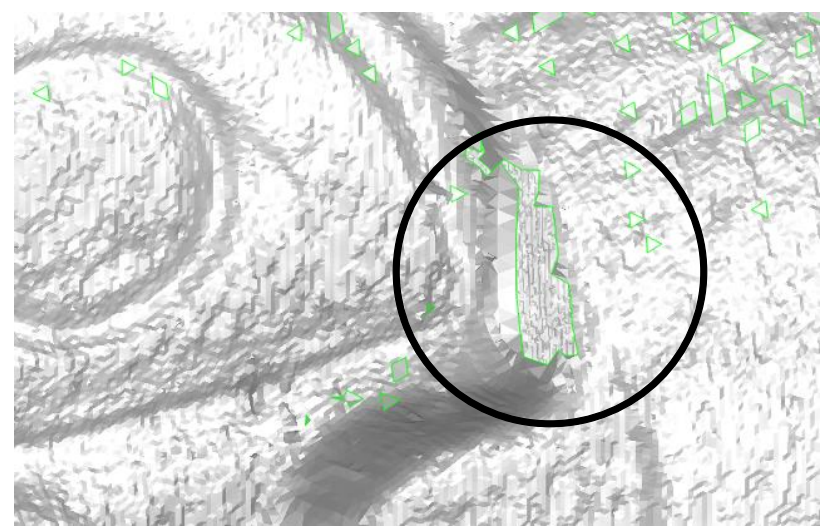

Figure 7. "Escultura 23". Missing polygons on the nose (circled).
Comparing the raw polygon models of both scanners after transformation, the most interesting fact is the difference in accuracy of detail reproduction. The mesh from Mantis does for example not represent the little teeth and only the big canine, whereas in the mesh from the $\mathrm{Z}+\mathrm{F}$ you can clearly distinguish them (fig. 8 and 9).

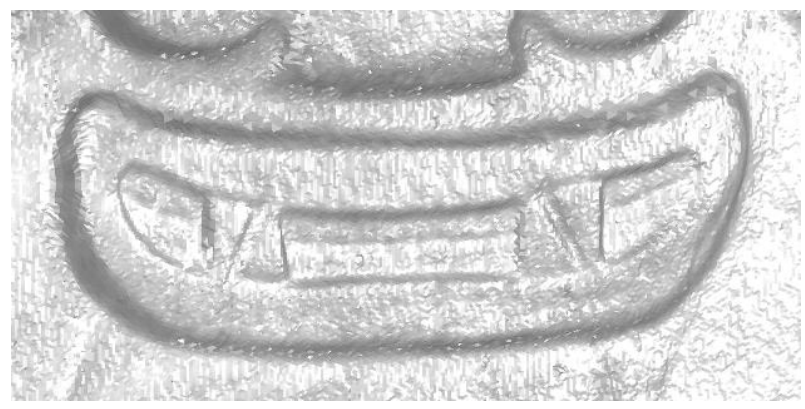

Figure 8. "Escultura 23". Detail of Z+F mesh showing the sculpture's mouth.

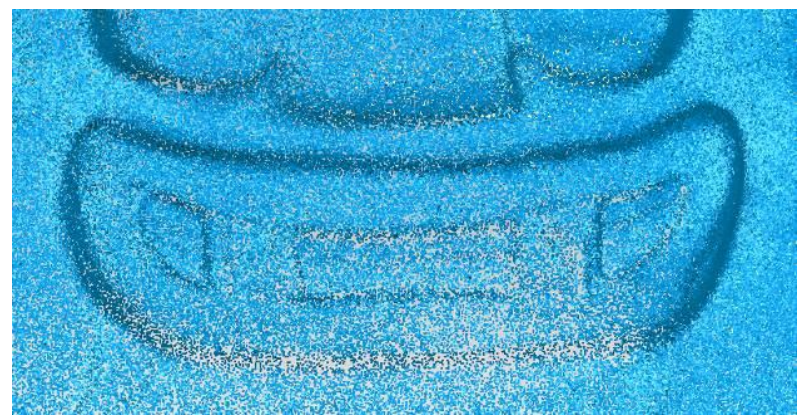

Figure 9. "Escultura 23". Detail of Mantis mesh showing the sculpture's mouth.

The mesh emanating from the Mantis (see also fig. 9) even though being much denser, when looking at the numbers, presents many little holes. This issue is surely due to wrong handling during the phase of data acquisition. Indeed, we were not free to use the equipment ourselves which was handled by the reseller's operator who was rather unexperienced in scanning cultural heritage. That is why for future projects, we hope to have more liberty in the usage of the scanners, so as to apprehend how to use them in the best appropriate way for cultural heritage applications. As we stated already earlier, the mesh from the $\mathrm{Z}+\mathrm{F}$ was much more detailed and presented only a few holes. In order to compare further both meshes, they have been aligned in Geomagic Studio using N-Point Alignment as well as the automated solution which uses the ICP algorithm (2). The manual alignment delivered more accurate results when checking for the deviation afterwards even if finding matching points in the mesh from the Mantis was not easy due to the numerous lacunae in the model. The calculated deviation was about $0.3 \mathrm{~mm}$ (RMS estimate). This means that the data from the Mantis hand held scanner was quite accurate and this reinforces as well our conclusion that the issue with this type of scanner lays in the correct data acquisition.

The better quality of the mesh from the terrestrial scanner was finally chosen to create the final model for "Escultura 23". After filling the lacunae and reworking the mesh, it has been exported as PLY and was textured in MeshLab. For the colour texture,

\footnotetext{
${ }^{2}$ Note that we reduced the triangle count to 2.5 million for the
} mesh from the Mantis. 
we used the eight photos that were saved in TIF format. We have chosen the open PLY format since we wish to insert the models in an online database. This standard should assure easy visualizing on different platforms. The final coloured model (fig. 10 ) is of decent quality, and fosters our hopes that the experience we gained in the process will allow us to obtain better results when acquiring the data of the next group of sculptures in San Agustín.

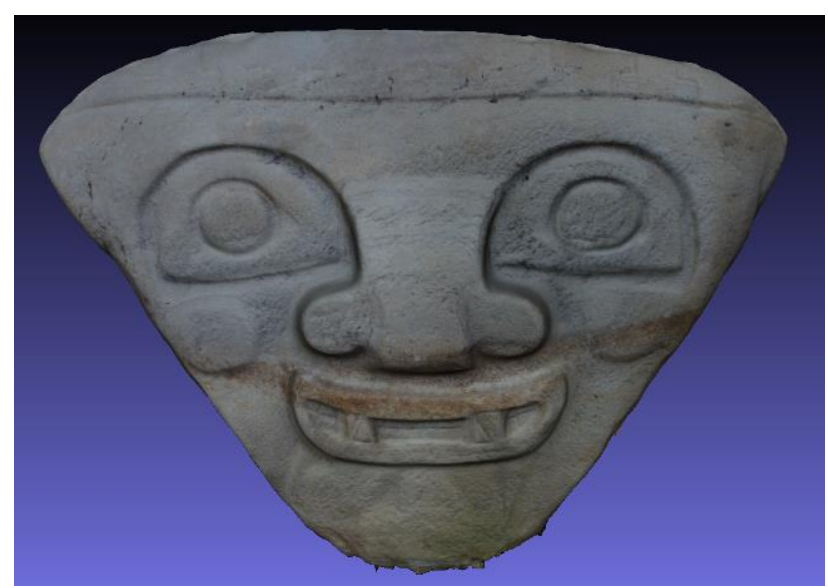

Figure 10. “Escultura 23”. Final colour textured model.

One of our scopes is surely to create models with a higher detail in which one can distinguish in the mesh the fine carved structures on the statues which are very important when it comes to conservational and to archaeological observations. If we want to preserve these sculptures, and not only "Escultura 23 ", in their present shape for future generations, it is critical to capture these details. Indeed, the constant exposure to weather is slowly but surely eroding the surface of these monuments and risks to wash away important art historical information. This becomes already obvious when looking at the figures 1 and 2; the surface of the sculpture seems smoother today when comparing two photographs that are separated by seven decades. Despite these observations, the 3D models exceed in quality the drawings and black-and-white photographs from the $20^{\text {th }}$ century. Certainly, there is still development needed until we will be able to use the models to display alterations as shown in figure 11 (drawing from 1974).
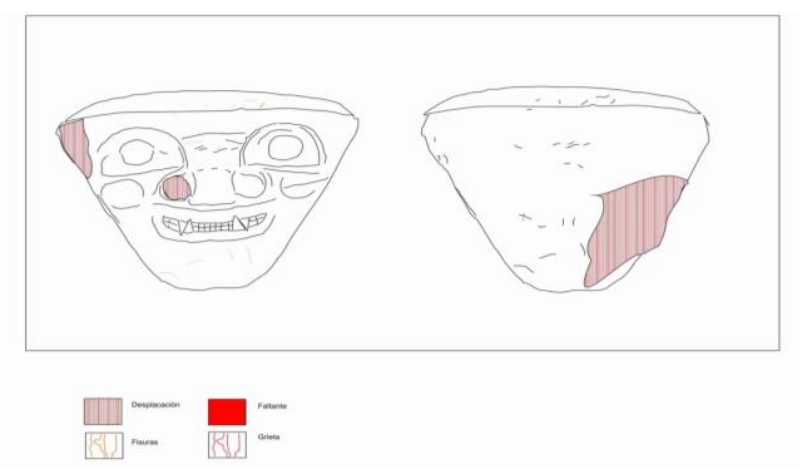

Figure 11. Alteration map of "Escultura 23". (Preuss, 1974, table 29-1).

\section{CONCLUSIONS}

The observations we made show the importance of the double approach chosen in this pilot project to elaborate a methodology for future endeavours. Indeed, the double datasets for some of the sculptures allowed us to rather choose the $\mathrm{Z}+\mathrm{F}$ data for the final models. In spite of the quite low deviation between meshes from Mantis and $\mathrm{Z}+\mathrm{F}$ and their high accuracy, we noticed many holes in the meshes and had to remodel manually smaller to bigger parts of the meshes in consequence. This does not allow an objective reconstruction of the statues which is necessary to be able to use the 3D models, either for exact calculations, or for conservational purposes, or even for archaeological studies (whereas the educational use is not altered as much). In conclusion, the particular environment and the particular kind of monuments want a specific approach and a greater experience which we hope to meet when scanning the next groups of statues in the upcoming months. We do also wish to continue with the double approach and think to integrate targets when scanning with the Mantis and reducing the number of frames. These conclusions show that our methodology was well established even if we lacked in experience when it comes to data acquisition.

This project has led to a first approach of the data acquisition technologies and post processing routines, resulting in quite a number of models. In the near future, we want to develop a solution that permits online access to this information. The sculptures have been geo-referenced so that the threedimensional models can be introduced in a database which we wish to render accessible mainly for research purposes. Furthermore, at present, we are working on a reconstruction and a simulation of the surrounding landscape, aiming at the realization of a virtual tour of different zones of the archaeological park as for example the "Fuente ceremonial del Lavapatas" (a ceremonial source).

The most important lesson learnt is however that future projects in Colombia would greatly profit of the creation of an interdisciplinary research group that develops virtualization projects and should ideally be supported by the institutions that deal with 3D data acquisition and modelling applied to cultural heritage. Indeed, the planning of the projects needs to be based on proven and tested methodologies in order to continue with subsequent works. Approaching new technologies of data acquisition allowed us to evaluate their application in special situations and circumstances. We deplore nonetheless that in Colombia the resellers impose that their equipment is used by their operators who do not possess any experience in data acquisition for complex objects such as the sculptures in San Agustín. This imposition as well as the lack of expertise and aid conditioned to a great extent the final outcome and the quality of the results.

\section{REFERENCES}

Pérez de Barradas J., 1943. Arqueología Agustíniana. Excavaciones realizadas de marzo a diciembre de 1937, Imprenta nacional, Bogotá, p. 49.

Preuss K. TH., 1974. Arte monumental prehistórico, Universidad nacional de Colombia, Bogotá, p. 78, table 29-1.

Sotomayor M. L. and Uribe M. V., 1987. Estatuaria del Macizo Colombiano, Colcultura, Bogotá, p. 4. 